Pacific Journal of Mathematics

THE EXTENSION OF EQUI-UNIFORMLY CONTINUOUS 


\title{
THE EXTENSION OF EQUI-UNIFORMLY CONTINUOUS FAMILIES OF MAPPINGS
}

\author{
RonNie LeVy AND Michael D. Rice
}

\begin{abstract}
In previous papers, we discussed the extension of uniformly continuous real-valued mappings from subspaces of metric spaces and the same question for mappings into certain Banach spaces, such as $c_{0}(I)$ and $l_{\infty}(I)$. Since the extension of uniformly continuous mappings into $l_{\infty}(I)$ is equivalent to the extension of equi-uniformly continuous point bounded families of real-valued mappings, it is natural to ask about the extension of equi-uniformly continuous families which are not necessarily point-bounded. The present paper investigates this extension property and several related questions concerning the extension of uniformly continuous mappings with values in uniformly discrete spaces.
\end{abstract}

I. Definitions and notation. Assume that $X$ and $Y$ are uniform spaces. Then $U(X, Y)$ denotes the family of uniformly continuous mappings from $X$ to $Y$. If $Y$ is the real line $R$, then $U(X, Y)$ will simply be denoted by $U(X)$. Assume that $S$ is a subset of $X$. Then the pair $(S, X)$ has the $Y$-extension property if every member of $U(S, Y)$ can be extended to a member of $U(X, Y)$. If $Y$ is the real line, we say that $S$ is $U$-embedded in $X$. It is a well-known theorem of Katětov that every bounded member of $U(S)$ can be extended to a member of $U(X)$.

If $D$ is a set, $l_{\infty}(D)$ is the set of all bounded real-valued functions on $D$ with the supremum metric. If the pair $(S, X)$ has the $l_{\infty}(D)$-extension property for every set $D$, we say that $(S, X)$ has the $l_{\infty}$-extension property. A straightforward translation shows that $(S, X)$ has the $l_{\infty}$-extension property if and only if every point-bounded equi-uniformly continuous subfamily of $U(S)$ can be extended to a point-bounded equi-uniformly continuous subfamily of $U(X)$. If every equi-uniformly continuous subfamily of $U(S)$ can be extended to an equi-uniformly continuous subfamily of $U(X)$, we say that $S$ is strongly $U$-embedded in $X$.

If $D$ is a set, $F(D, R)$ denotes the family of all real-valued functions on $D$ with the metric defined by $d(f, g)=\sup \{|f(x)-g(x)| \wedge 1: x \in$ $D\}$. In addition, we define $\|f-g\|=\sup \{|f(x)-g(x)|: x \in D\}$, allowing the possible value $+\infty$. Using the definition of the metric space $F(D, R)$, one can show (i) that $l_{\infty}(D)$ is a uniform subspace of $F(D, R)$, and (ii) that $S$ is strongly $U$-embedded in $X$ if and only if the pair $(S, X)$ has the $F(D, R)$-extension property for every set $D$. 
A uniform space is uniformly discrete if $\{\{p\}: p \in D\}$ is a uniform cover of $D$. A uniform partition of a uniform space is a uniform cover $\left\{U_{j}\right\}$ consisting of disjoint sets; equivalently, the cover is a uniform partition if there exists a uniformly continuous pseudometric $d$ and an $r>0$ such that $d\left(U_{j}, U_{k}\right) \geq r$ for $j \neq k$. It is routine to verify that for any uniformly discrete space $D$ of power $m$, the pair $(S, X)$ has the $D$-extension property if and only if for each uniform partition $\mathscr{U}$ of $S$ of power at most $m$, there exists a uniform partition $\mathscr{V}$ of $X$ such that $\mathscr{V} \mid S=\{V \cap S: V \in \mathscr{V}\}$ refines $\mathscr{U}$. Finally, we note that if $(S, X)$ has the $\{0,1\}$-extension property $(m=2)$, then $(S, X)$ has the $\{0,1, \ldots, n\}$-extension property for every finite $n$.

II. General results. We will see in section four that there are some rather striking differences between extension properties in metric spaces and in more general uniform spaces. However, there are also some results which hold in the more general setting of uniform spaces. Here we will discuss some of these properties.

2.1. Lemma. If $|D|=m \geq \boldsymbol{\aleph}_{0}$, then the uniformly discrete space of power $2^{m}$ is a uniform retract of $F(D, R)$.

Proof. Without loss of generality, we will assume that $D=A \times N$, where $|A|=m$. For $B \subseteq A$, define $f_{B}: D \rightarrow R$ by the rule

$$
f_{B}(x, n)= \begin{cases}n & \text { if } x \in B \\ 0 & \text { if } x \notin B .\end{cases}
$$

Notice that if $B \neq C$ and $x \in[(B-C) \cup(C-B)]$, then

$$
\left|f_{B}(x, n)-f_{C}(x, n)\right|=n \text {. }
$$

Hence

$$
B \neq C \text { implies }\left\|f_{B}-f_{C}\right\|=+\infty .
$$

Let $S=\left\{f_{B}: B \subseteq A\right\}$. Then $|S|=2^{|A|}=2^{m}$ and it follows from $(*)$ that $S$ is a uniformly discrete subset of $F(D, R)$. Define $r: F(D, R) \rightarrow S$ by the rule

$$
r(g)= \begin{cases}f_{B} & \text { if }\left\|g-f_{B}\right\|<+\infty, \\ f_{A} & \text { otherwise. }\end{cases}
$$

It follows from $(*)$ that $r$ is a well-defined uniform retraction.

\section{REMARKS.}

1. In the preceding proof, $f_{\varnothing}$ is the constant function with value zero. Hence $\left\|g-f_{\varnothing}\right\|<+\infty$ is equivalent to the statement that $g$ is a bounded 
mapping, so $l_{\infty}(D)=r^{-1}\left(f_{\varnothing}\right)$. Since $l_{\infty}(D)$ is one member of a uniform partition, it follows that $l_{\infty}(D)$ is also a uniform retract of $F(D, R)$.

2. Lemma 2.1 does not hold if $D$ is a finite set since in this case, $F(D, R)$ is a connected space.

2.2. THEOREM. The following statements are equivalent for a subset $S$ of the uniform space $X$.

(i) $S$ is strongly $U$-embedded in $X$.

(ii) $S \times D$ is $U$-embedded in $X \times D$ for every uniformly discrete space D.

(iii) The pair $(S, X)$ has the $l_{\infty}$-extension property and the $D$-extension property for every uniformly discrete space $D$.

Proof. The equivalence of (i) and (ii) is a consequence of the following fact. If $Y$ is a uniform space and $D$ is uniformly discrete, then the mapping $f: Y \times D \rightarrow R$ is uniformly continuous if and only if the family of mappings $\{f(-, k)\}: Y \rightarrow R$ is equi-uniformly continuous.

(i) $\rightarrow$ (iii): Since $S$ is $U$-embedded in $X,(S, X)$ has the $l_{\infty}(D)$-extension property for every finite set $D$, for in this case, $l_{\infty}(D)$ is uniformly equivalent to $R^{D}$. If $D$ is infinite, then by Remark 1 above, $l_{\infty}(D)$ is a uniform retract of $F(D, R)$. Therefore, since $(S, X)$ has the $F(D, R)$-extension property, it also has the $l_{\infty}(D)$-extension property. In the same vein, by $2.1, D$ is a uniform retract of $F(D, R)$, so $(S, X)$ has the $D$-extension property.

(iii) $\rightarrow$ (i): Assume that $\left\{f_{k}: k \in K\right\}$ is an equi-uniformly continuous subfamily of $U(S)$. There exists a uniform cover $\mathscr{U}$ of $S$ such that for every $U$ in $\mathscr{U},\{x, y\} \subseteq U$ implies $\left|f_{k}(x)-f_{k}(y)\right|<1$ for every $k \in K$. Define the following equivalence relation $\sim$ on $S$ :

$x \sim y$ if and only if $x \in \operatorname{St}^{n}(y, \mathscr{U})$ for some $n=1,2, \ldots$

Then $\mathscr{J}=\{E: E$ is an equivalence class $\}$ is a uniform partition of $S$ (since $\mathscr{U}<\mathscr{J})$.

For each equivalence class $E$, fix an element $p_{E}$ in $E$ and for each $k$ in $K$, define a mapping $g_{k}: S \rightarrow R$ in the following manner:

$$
g_{k}(x)=f_{k}(x)-f_{k}\left(p_{E}\right) \text { if } x \in E .
$$

We claim that $\left\{g_{k}\right\}$ is an equi-uniformly continuous point-bounded family. Since $\mathscr{J}_{\text {is }}$ a uniform partition and $\left\{f_{k}\right\}$ is equi-uniformly continuous, $\left\{g_{k}\right\}$ is equi-uniformly continuous. To show that $\left\{g_{k}\right\}$ is pointbounded, suppose that $x$ is a member of the equivalence class $E$. Since $x \sim p_{E}$, there exist finitely many members $U_{1}, U_{2}, \ldots, U_{n}$ of $\mathscr{U}$ such that 
$x \in U_{1}, p_{E} \in U_{n}$, and $U_{m} \cap U_{m+1} \neq \varnothing$ for $1 \leq m \leq n-1$. Then it is straightforward to verify that for every $k$,

$$
\left|g_{k}(x)\right| \leq\left|g_{k}(x)-g_{k}\left(p_{E}\right)\right|+\left|g_{k}\left(p_{E}\right)\right| \leq n+0=n .
$$

By (iii), $\left\{g_{k}\right\}$ extends to an equi-uniformly continuous family $\left\{G_{k}\right\}$ $\subseteq U(X)$ and the partition $\mathscr{J}$ extends to a uniform partition $\mathscr{P}$ of $X$. Define $h_{k}: X \rightarrow R$ by $h_{k}(x)=f_{k}\left(p_{E}\right)$ if $x \in P \in \mathscr{P}$, where $P \cap S=E$, and $h_{k}(x)=0$ if $P \cap S=\varnothing$. Then $\left\{h_{k}\right\}$ is an equi-uniformly continuous family and the equi-uniformly continuous family $\left\{G_{k}+h_{k}\right\}$ is an extension of $\left\{f_{k}\right\}$.

A uniform space is uniformly connected if there is no uniform partition with more than one member; equivalently, the space $X$ is uniformly connected if every uniformly continuous mapping from $X$ into a uniformly discrete space is constant. In particular, if $X$ has a ce nected topology, $X$ is uniformly connected.

2.3. Corollary. A uniformly connected subset $S$ of $X$ is strongly $U$-embedded if and only if the pair $(S, X)$ has the $l_{\infty}$-extension property.

III. Results for metric spaces. In this section, we will strengthen Theorem 2.2 for metric spaces by weakening the $D$-extension property in condition (iii). In addition, we will characterize the strongly $L$-embedded subsets of normed linear spaces.

Assume that $(X, d)$ is a metric space. Given $r>0$, the points $x$ and $y$ are $r$-linked (in $X$ ) with $n$-links if there is a finite sequence of points $x=x_{0}, x_{1}, \ldots, x_{n}=y$, such that $d\left(x_{k}, x_{k+1}\right) \leq r$ for $k=0,1, \ldots, n-1$. If there exists such a sequence, we simply say that $x$ and $y$ are $r$-linked and write $x \stackrel{r}{\sim} y$. In this case, we define

$$
n_{r}(x, y)=\min \{n: x \text { and } y \text { are } r \text {-linked with } n \text {-links }\} \text {. }
$$

For each $r>0, \stackrel{r}{\sim}$ is an equivalence relation and the resulting collection of equivalence classes is denoted $\mathscr{J}(r)$. Since every sphere of radius $r$ is contained in some equivalence class, it follows that $\mathscr{J}(r)$ is a uniform partition of $X$.

3.1. Lemma. Assume that $S$ is a subset of the metric space $(X, d)$ and $\mathscr{P}$ is a uniform partition of $S$. If there exists an $r>0$ such that no two distinct elements of $\mathscr{P}$ contain points which are r-linked in $X$, then $\mathscr{P}$ extends to a uniform partition of $X$. 
Proof. The assumptions guarantee that $\mathscr{J}(r) \mid S<\mathscr{P}$, so the conclusion follows at once.

3.2. TheOREM. Assume that $S$ is a subset of the metric space $(X, d)$. Then $S$ has the $\{0,1\}$-extension property if and only if $S$ has the D-extension property for every uniformly discrete space $D$.

Proof. Assume that $S$ has the $\{0,1\}$-extension property and suppose that $\mathscr{P}$ is a uniform partition of $S$ which does not extend to a uniform partition of $X$. We first establish the following result:

(*) If $\mathscr{F}$ is a finite subset of $\mathscr{P}$, then the uniform partition $\mathscr{P}-\mathscr{F}$ of $S-(\bigcup \mathscr{F})$ does not extend to a uniform partition of $X$.

Since $S$ has the $\{0,1\}$-extension property, every finite uniform partition of $S$ extends to a uniform partition of $X$. If $\mathscr{P}-\mathscr{F}$ extends to a uniform partition $\breve{\mathscr{P}}$ of $X$ and $\breve{F}$ is a uniform partition of $X$ which extends the finite uniform partition $\mathscr{F} \cup\{\cup(\mathscr{P}-\mathscr{F})\}$ of $S$, then $\check{\mathscr{P}} \wedge \check{\mathscr{F}}$ extends $\mathscr{P}$, which is a contradiction. Therefore, $(*)$ is established.

We will now use 3.1 and $(*)$ to inductively define two sequences of members of $\mathscr{P}$. By 3.1, first choose distinct members $P(1)$ and $Q(1)$ of $\mathscr{P}$ such that there exist points $x_{1}$ in $P(1)$ and $y_{1}$ in $Q(1)$ with $x_{1} \sim y_{1}$. Inductively, by 3.1 and $(*)$, choose distinct members $P(n)$ and $Q(n)$ of $\mathscr{P}-(\{P(k): k<n\} \cup\{Q(k): k<n\})$ such that there exist points $x_{n}$ in $P(n)$ and $y_{n}$ in $Q(n)$ with $x_{n}{ }^{1 / n} y_{n}$.

Let $A=\bigcup\{P(n): n=1,2, \ldots\}$ and $B=S-A$. Then $\{A, B\}$ is a uniform partition of $S$, so it extends to a uniform partition $\{\hat{A}, \hat{B}\}$ of $X$. Choose an integer $n$ such that $d(\hat{A}, \hat{B})>1 / n$. Then no point in $\hat{A}$ can be $1 / n$-linked to any point in $\hat{B}$, which contradicts the fact that $x_{n} \sim y_{n}$. Therefore, every uniform partition of $S$ extends to a uniform partition of $X$, so $(S, X)$ has the $D$-extension property for every uniform discrete space $D$.

REMARK. In $\S 4$ we will see that the analogue of 3.2 is not valid for general uniform spaces.

3.3. THEOREM. The following statements are equivalent for a subset $S$ of the metric space $X$.

(i) $S$ is strongly $U$-embedded in $X$.

(ii) The pair $(S, X)$ has the $l_{\infty}$-extension property and the $\{0,1\}$-extension property. 
Here is another proof of Theorem 3.2. Assume that $(X, d)$ is a metric space and $d_{0}$ denotes the uniformity on $X$ generated by the $d$-uniform partitions. It is routine to show that $d_{0}$ has the countable basis $\{g(1 / n)$ : $n=1,2, \ldots\}$, so $d_{0} \mid S$ also has a countable basis and is therefore proximally fine (that is, the largest uniformity in its proximity class). If $S$ has the $\{0,1\}$-extension property, the uniformity $u$ on $S$ generated by the $d$-uniform partitions has the same proximity class as $d_{0} \mid S$. Therefore, $u \subseteq d_{0} \mid S$, which says that $(S, X)$ has the $D$-extension property for every uniformly discrete space $D$.

We will now turn our attention to normed linear spaces. Theorem 2 in $[\mathbf{L R}]_{1}$ establishes that a subset $S$ of the normed linear space $(B,\|\|)$ is $U$-embedded if and only if every uniformly continuous mapping $f: S \rightarrow R$ is Lipschitz for large distances: for every $r>0$, there exists a constant $K_{r}$ such that $\|x-y\|>r$ implies $|f(x)-f(y)| \leq K_{r}\|x-y\|$. In addition, Theorem 3.1 in [LR $]_{3}$ establishes that $S$ is $U$-embedded in $B$ if and only if the pair $(S, B)$ has the $l_{\infty}$-extension property. Using this result with 3.3, we can establish the following result.

3.4. TheOREM. $A$ subet $S$ of the normed linear space $B$ is strongly $U$-embedded if and only if $S$ is $U$-embedded and uniformly connected.

Proof. Based on the result cited above and 3.3, we need only show that strongly $U$-embedded sets are uniformly connected. However, if $\{C, D\}$ is a two element uniform partition of $S$, then by either 2.2 or 3.3, $\{C, D\}$ can be extended to a uniform partition of $B$, which is a contradiction, since $B$ is connected. Therefore, $S$ is uniformly connected.

3.5. TheOrem. $A$ subset $S$ of the normed linear space $B$ is strongly $U$-embedded if and only if $S \times B$ is $U$-embedded in $B \times B$.

Proof. Assume that $S$ is strongly $U$-embedded in $X$. We will show that every uniformly continuous mapping $f: S \times B \rightarrow R$ is Lipschitz for large distances. Then it will follow from the result cited before 3.4 that $S \times B$ is $U$-embedded in $B \times B$. For each $s$ in $S$, define $g_{s}: B \rightarrow R$ by $g_{s}(x)=$ $f(s, x)$ and for each $x$ in $B$, define $h_{x}: S \rightarrow R$ by $h_{x}(s)=f(s, x)$. Since $f$ is uniformly continuous, each family $\left\{g_{s}\right\}$ and $\left\{h_{x}\right\}$ is equi-uniformly continuous, so by assumption, $\left\{h_{x}\right\}$ can be extended to an equi-uniformly continuous subfamily $\left\{\breve{h}_{x}\right\}$ of $U(B)$. Then by the remark after Lemma 2 in $[\mathbf{L R}]_{1}$, there exists two families of Lipschitz mappings $\left\{l_{s}\right\}$ and $\left\{m_{x}\right\}$ 
on $B$, and constants $K$ and $L$, such that

(i) $\left|l_{s}(x)-l_{s}(y)\right| \leq K\|x-y\|$ for every $s$ in $S, x$ and $y$ in $B$, $\left|m_{x}(p)-m_{x}(q)\right| \leq L\|p-q\|$ for every $x, p$ and $q$ in $B$

(ii) $\left\|l_{s}-g_{s}\right\| \leq 1$ for every $s$ in $S$,

$\left\|m_{x}-h_{x}\right\| \leq 1$ for every $x$ in $B$.

Assume that $A=(s, x)$ and $C=(t, y)$ are two elements of $S \times B$ and let $P=(s, y)$. Then

$$
\begin{aligned}
\mid f(A)- & f(C)|\leq| f(A)-f(P)|+| f(P)-f(C) \mid \\
= & |f(s, x)-f(s, y)|+|f(s, y)-f(t, y)| \\
= & \left|g_{s}(x)-g_{s}(y)\right|+\left|h_{y}(s)-h_{y}(t)\right| \\
\leq & \left|g_{s}(x)-l_{s}(x)\right|+\left|l_{s}(x)-l_{s}(y)\right| \\
& +\left|l_{s}(y)-g_{s}(y)\right|+\left|h_{y}(s)-m_{y}(s)\right| \\
& +\left|m_{y}(s)-m_{y}(t)\right|+\left|m_{y}(t)-h_{y}(t)\right| \\
\leq & K\|x-y\|+L\|s-t\|+4 \quad(\text { by }(\mathrm{i}) \text { and (ii)) } \\
\leq & 2(K+L)\|(s, x)-(t, y)\|_{B \times B}+4 \\
\leq & 2(K+L)\|A-C\|+(4 / r)\|A-C\| \quad \text { (if }\|A-C\|>r) \\
= & (2 K+2 L+4 / r)\|A-C\| .
\end{aligned}
$$

Hence $f$ is Lipschitz for large distances, so we have established that $S \times B$ is $U$-embedded in $B \times B$.

Now assume that $S \times B$ is $U$-embedded in $B \times B$. We will show that $S$ is a uniformly connected $U$-embedded subset of $B$; then by $3.5, S$ will be strongly $U$-embedded in $B$. To show that $S$ is $U$-embedded in $B$, let $f$ be a member of $U(S)$ and define $f: S \times B \rightarrow R$ by $f(s, x)=f(s)$. $f$ is uniformly continuous, so by assumption it extends to a member $F$ of $U(B \times B)$. Then the mapping $F: B \rightarrow R$ defined by $F(x)=F(x, 0)$ is a uniformly continuous extension of $f$. Finally, if $S$ is not uniformly connected, there exists a non-trivial uniform partition $\{C, D\}$ of $S$. Define the mapping $f: S \times B \rightarrow R$ by $f(s, x)=0$ if $s \in C$ and $f(s, x)=$ $\|x\|$ if $s \in D$. Since $\{C, D\}$ is a uniform partition, $f$ is uniformly continuous, so by assumption it extends to a uniformly continuous mapping $\check{f}$ : $B \times B \rightarrow R$. Fix points $c$ in $C$ and $d$ in $D$. Choose $r>0$ such that $\|s-t\|<r$ implies $|\check{f}(s, x)-\check{f}(t, x)| \leq 1$ for all $x$ in $B$. Since $c$ and $d$ are $r$-linked in $B$, it follows that $\|x\|=|\check{f}(c, x)-\check{f}(d, x)| \leq n_{r}(c, d)$ for every $x$ in $B$, which is a contradiction. Therefore, $S$ is uniformly connected and the proof is complete. 
3.6. THEOREM. The following statements are equivalent for a subset $S$ of the normed linear space $(B,\|\|)$.

(i) $S \times S$ is $U$-embedded in $B \times B$.

(ii) $S \times S$ is $U$-embedded in $S \times B$.

(iii) Either $S$ is strongly $U$-embedded in $B$ or every member of $U(S)$ is bounded.

Proof. Clearly, (i) $\rightarrow$ (ii).

(ii) $\rightarrow$ (iii): A proof similar to the one given in 3.5 shows that $S$ is $U$-embedded in $B$. Therefore, to establish (iii), by 3.4 it suffices to show that if there exists an unbounded uniformly continuous mapping $h$ : $S \rightarrow R$, then $S$ is uniformly connected. Assume that $\{C, D\}$ is a non-trivial uniform partition of $S$ and define the mapping $f: S \times S \rightarrow R$ by the rule

$$
f(x, y)= \begin{cases}|h(x)| & \text { if } y \in C \\ 0 & \text { if } y \in D\end{cases}
$$

$f$ is uniformly continuous, so by (ii), it extends to a uniformly continuous mapping $\check{f}: S \times B \rightarrow R$. Choose points $x_{1}, x_{2}, \ldots$ in $S$ such that $\left|h\left(x_{k}\right)\right|$ $>k$ for $k=1,2, \ldots$ and for each $n=1,2, \ldots$ define $g_{n}: B \rightarrow R$ by $g_{n}(y)=\check{f}\left(x_{n}, y\right)$. Fix points $c$ in $C$ and $d$ in $D$. Since $\breve{f}$ is uniformly continuous, the family $\left\{g_{n}\right\}$ is equi-uniformly continuous, so by $[\mathbf{L R}]_{1}$, for any $r>0$, there exists a constant $K_{r}$ such that $\|x-y\|>r$ implies $\left|g_{n}(x)-g_{n}(y)\right| \leq K_{r}\|x-y\|$ for $n=1,2, \ldots$ Therefore, there exists a constant $K$ such that $\left|g_{n}(c)-g_{n}(d)\right| \leq K\|c-d\|$ for every $n$. Since $d \in D, g_{n}(d)=f\left(x_{n}, d\right)=0$; hence

$$
n \leq\left|h\left(x_{n}\right)\right|=\left|f\left(x_{n}, c\right)\right| \leq\left|g_{n}(c)\right| \leq K\|c-d\| \text { for every } n,
$$

which is a contradiction. Therefore, $S$ is uniformly connected, so (iii) is established.

(iii) $\rightarrow$ (i): If $S$ is strongly $U$-embedded in $B$, the proof given in 3.5 shows that $S \times S$ is $U$-embedded in $B \times B$. If every member of $U(S)$ is bounded, the same property holds for $S \times S$. (Assume that $f: S \times S \rightarrow R$ is a uniformly continuous mapping. For each $s$ in $S$, define $g_{s}: S \rightarrow R$ by $g_{s}(t)=f(s, t)$. Since $f$ is uniformly continuous, the family $\left\{g_{s}\right\}$ is equiuniformly continuous, and since for every $t$, the mapping $f(-, t)$ is bounded, the family $\left\{g_{s}\right\}$ is also point-bounded. Therefore, the mapping $h: S \rightarrow R$ defined by

$$
h(t)=\sup \left\{\left|g_{s}(t)\right|: s \in S\right\}
$$


is uniformly continuous and hence bounded, so $f$ is also bounded.) Since every member of $U(S \times S)$ is bounded, it follows from the Katětov extension theorem that $S \times S$ is $U$-embedded in $B \times B$, so the proof of (i) is complete.

IV. Uniformly discrete subsets. In this section we will consider the special situation when $S$ is a uniformly discrete subset of $X$. In particular, we will present several examples which show that 3.2 and 3.3 cannot be established for arbitrary uniform spaces.

4.1. Proposition. Assume that $S$ is a uniformly discrete subset of the uniform space $X$. Then $S$ is strongly $U$-embedded in $X$ if and only if $S$ is a uniform retract of $X$.

Proof. If $S$ is strongly $U$-embedded in $X$, then by $2.2, S$ has the $D$-extension property for every uniformly discrete $D$. Hence the identity mapping $i: S \rightarrow S$ can be extended to a uniformly continuous mapping $r$ : $X \rightarrow S$, so $S$ is a uniform retract of $X$. Conversely, if $r: X \rightarrow S$ is a uniform retraction and $\mathscr{F}=\left\{f_{k}\right\} \subseteq U(S)$ is an equi-uniformly continuous family, then $\left\{f_{k} \circ r\right\}$ is an equi-uniformly continuous family which extends $\mathscr{F}$.

4.2. Proposition. Assume that $S$ is a uniformly discrete subset of the metric space $X$.

(i) The pair $(S, X)$ has the $\{0,1\}$-extension property if and only if $S$ is a uniform retract of $X$.

(ii) $S$ is $U$-embedded in $X$ if and only if some cofinite subset of $S$ is a uniform retract of $X$.

Proof. (i) follows from 3.2 and 4.1.

(ii): Assume that no cofinite subset of $S$ is a uniform retract of $X$. Using 3.1, with $\mathscr{P}$ the collection of singleton sets of $S$, there exist distinct points $x_{1}$ and $y_{1}$ in $S$ such that $x_{1} \sim y_{1}$. Since $S-\left\{x_{1}, y_{1}\right\}$ is not a uniform retract of $X$, by 3.1 there exist distinct points $x_{2}$ and $y_{2}$ in $S-\left\{x_{1}, y_{1}\right\}$ such that $x_{2} \stackrel{1 / 2}{\sim} y_{2}$. Inductively, there exist distinct points $x_{n}$ and $y_{n}$ in $S-\left\{x_{k}, y_{k}: k<n\right\}$ such that $x_{n}{ }^{1 / n} y_{n}$. We will use the sequences $\left\{x_{k}\right\}$ and $\left\{y_{k}\right\}$ to show that $S$ is not $U$-embedded in $X$. Define the mapping $f: S \rightarrow R$ by

$$
f\left(x_{k}\right)=\left[n_{1 / k}\left(x_{k}, y_{k}\right)\right]^{2} \quad \text { for } k=1,2, \ldots
$$


and $f(x)=0$ for $x \in S-\left\{x_{k}\right\}$. Since $S$ is uniformly discrete, $f$ is uniformly continuous, but it cannot be extended to a uniformly continuous mapping on $X$ since $x_{k} \sim y_{k}$ for $k=1,2, \ldots$ Therefore, $S$ is not $U$-embedded in $X$.

Conversely, suppose that $F$ is a finite subset of $S$ such that $S-F$ is a uniform retract of $X$. Then $S-F$ is $U$-embedded in $X$ and every real-valued function on $S$ whose restriction to $S-F$ is bounded, is itself bounded. It follows from Theorem 4.3 in $[\mathbf{L R}]_{2}$ that $S$ is $U$-embedded in $X$.

4.3. COROllary. If a metric space contains an infinite uniformly discrete $U$-embedded subset, then it admits an infinite uniform partition.

REMARKS. 1. 4.2(ii) and 4.3 are not valid for arbitrary uniform spaces. For example, if $X$ is the real line with the fine uniformity and $S$ is the set of natural numbers, then $S$ is $C$-embedded, and hence $U$-embedded in $X$, but the conclusions of 4.2 (ii) and 4.3 fail since $X$ is connected.

2. Example 4.4 below shows that 4.2 (i) is not valid for arbitrary uniform spaces.

3. The proof of Lemma 1.5 in [CI] establishes that if every uniformly discrete subset of a metric space is $U$-embedded, then every subspace is $U$-embedded. (Uniform spaces with this property are called real-extension or RE spaces.) Thus, by 4.2, a metric space $X$ is an RE space if and only if every uniformly discrete subspace of $X$ contains a cofinite subset which is a uniform retract of $X$.

4. The proof of necessity in 4.2(ii) actually shows that the phrase " $S$ is $U$-embedded in $X$ " can be replaced by the phrase " $(S, X)$ has the $l_{\infty}$-extension property" (or the $B$-extension property for any normed linear space $B$ ) and the result will still be valid. In particular, uniformly discrete $U$-embedded subsets of metric spaces have the $l_{\infty}$-extension property. The authors do not know whether every $U$-embedded subset of a metric space (or even a uniform space) has the $l_{\infty}$-extension property.

We will now present some examples which show that 3.2 and 4.2(i) are not valid in arbitrary uniform spaces. If $m$ is a cardinal number, $D(m)$ will denote the uniformly discrete space of power $m$.

4.4. ExAmple. Assume $m \geq \boldsymbol{\aleph}_{0}$. There exists a uniform space $X$ which contains a uniformly discrete subset $S$ of power $m$ such that

(a) For each cardinal $n<m,(S, X)$ has the $D(n)$-extension property.

(b) $(S, X)$ has the $l_{\infty}$-extension property. 
(c) $X$ admits no uniform partition of power $m$, so $(S, X)$ does not have the $D(m)$-extension property.

Construction. Let $X=D(m) \times[0,1]$ with the usual product topology. For each partition $\mathscr{P}$ of $D(m)$ such that $|\mathscr{P}|<m$ and each $r>0$, define

$$
A(\mathscr{P}, r)=\{P \times([0, r) \cap[0,1]): P \in \mathscr{P}\} .
$$

Let $u$ be the uniformity on $X$ generated by covers of the form $A(\mathscr{P}, r) \cup \mathscr{U}$, where $|\mathscr{P}|<m$ and $\mathscr{U}$ is an open cover of $D(m) \times(0,1]$. (Using the fact that every open cover of $D(m) \times(0,1]$ has an open star-refinement, one can verify that this family of covers generates a uniformity.) The subset $S=D(m) \times\{1\}$ is uniformly discrete since $(A(\mathscr{P}, 1) \cup \mathscr{U}) \mid S$ consists of singleton sets, where $\mathscr{P}=\{D(m)\}$ and $\mathscr{U}=\{\{x\} \times(0,1]: x \in D(m)\}$. In addition, if $\mathscr{P}$ is a partition of $S$ of power $<m$, then $A(\mathscr{P}, 2)$ is a uniform partition of $X$ which extends $\mathscr{P}$. Hence (a) is satisfied.

To establish (b), assume that $f: S \rightarrow B$ is any mapping with values in the Banach space $B$. Define a mapping $F: X \rightarrow B$ in the following manner: $F(x, 1)=f(x, 1)$ for $x \in D(m), F(x, r)=0$ if $0 \leq r \leq 1 / 2$, and $F$ linear on the segment between $(x, 1 / 2)$ and $(x, 1)$. Clearly, $F$ is uniformly continuous and extends $f$, so (b) is satisfied.

Finally, to establish (c), suppose that $\mathscr{S}$ is a uniform partition of $X$ which is refined by the uniform cover $A(\mathscr{P}, r) \cup \mathscr{U}$, where $\mathscr{P}=\left\{P_{t}\right\}$ has power $<m$ and $\mathscr{U}$ is an open cover of $D(m) \times(0,1]$. Assume that

$$
P_{t} \times([0, r) \cap[0,1]) \subseteq S_{t},
$$

for some $S_{t}$ in $\mathscr{S}$. If $r>1$, then the family $\left\{S_{t}\right\}$ covers $X$, so $|\mathscr{S}|<m$. If $0<r \leq 1$, then every point $(x, s)$, where $r \leq s \leq 1$, belongs to some $U$ in $\mathscr{U}$, which in turn, is connected by a finite chain of sets in $\mathscr{U}$ to a set of the form $P_{t} \times[0, r)$. Since $\mathscr{S}$ is a partition, $(x, r)$ belongs to $S_{t}$, so once again, $\left\{S_{t}\right\}$ covers $X$ and $|\mathscr{S}|<m$.

If $m=\aleph_{0}$, the preceding example shows that both 3.2 and 4.2(i) fail for arbitrary uniform spaces. A more dramatic example is given in the next construction.

4.5. EXAMPLE. There exists a locally compact abelian group $X$ with the usual group uniformity and an infinite uniformly discrete subset $S$ of $X$ such that

(a) $(S, X)$ has the $\{0,1\}$-extension property.

(b) $X$ admits no infinite uniform partition.

Construction. Let $Z$ denote the additive group of integers and let $Z_{2}=Z / 2 Z$. Let $P$ denote the power set of $Z$ and define the group 
$X=Z_{2}^{P} \times R$ with the usual product uniformity. Since $Z_{2}^{P}$ is compact and $R$ is connected, $X$ admits no infinite uniform partitions, so (b) is satisfied. To define the subset $S$, for each $A \subseteq Z$, let $p_{A}: X \rightarrow Z_{2}$ denote the projection mapping corresponding to the coordinate $A: p_{A}(f, x)=f(A)$. For $n \in Z$, define an element $g_{n}$ in $Z_{2}^{P}$ by the rule $g_{n}(A)=1$ if $n \in A$, $g_{n}(A)=0$ if $n \notin A$. Let $S=\left\{\left(g_{n}, n\right): n \in Z\right\} . S$ is uniformly discrete since the mapping $Z \rightarrow S$ defined by $n \nrightarrow\left(g_{n}, n\right)$ is an onto uniform equivalence. To establish (a), for each subset $T$ of $S$, we must find a uniform partition $\{C, D\}$ of $X$ such that $(*): T \subseteq C$ and $S-T \subseteq D$. Set $\check{T}=\left\{n:\left(g_{n}, n\right) \in T\right\}$ and define $C=p_{\breve{T}}^{-1}(1)$ and $D=p_{\breve{T}}^{-1}(0)$. Clearly, $\{C, D\}$ is a uniform partition of $X$, so we only have to verify $(*)$. If $\left(g_{n}, n\right) \in T$, then $n \in \check{T}$, so $p_{\check{T}}\left(g_{n}, n\right)=g_{n}(\check{T})=1$ implies that $\left(g_{n}, n\right) \in$ $C$; hence $T \subseteq C$. Similarly, if $\left(g_{n}, n\right) \in S-T$, then $n \notin \check{T}$, so $p_{\breve{T}}\left(g_{n}, n\right)$ $=g_{n}(\check{T})=0$ implies that $\left(g_{n}, n\right) \in D$; hence $S-T \subseteq D$, so the proof is complete.

REMARKs. 1. Assertions (a) and (b) in the preceding example are still valid if $X$ is given the fine uniformity instead of the group uniformity; hence 3.2 and 4.2(i) are not valid for the fine uniformity on special topological spaces. (See remark 3 below.)

2. We do not know an example of a group $X$ which contains an infinite discrete subgroup $S$ such that properties (a) and (b) hold. In the preceding construction, one can show that if $S$ is replaced by the subgroup $G$ of $X$ generated by $S$, then there exist finite uniform partitions of $G$ which do not extend to uniform partitions of $X$. This follows from the fact that $(0, n)$ belongs to $G$ if (and only if, although this is not relevant) $n$ is even.

3. Finally, we remark that Example 4.5 has some interesting properties as a topological space. $X$ is a locally compact, $\sigma$-compact, separable, homogeneous, Hausdorff space which contains a closed (and hence $C$-embedded) countable discrete subset such that every finite-valued function extends to a continuous finite-valued function on the superspace, but no $N$-valued function with infinite image extends to a continuous function with values in $N$.

\section{REFERENCES}

[CI] H. H. Corson and J. R. Isbell, Some properties of strong uniformities, Quart. J. Math. Oxford (2), 11 (1960), 17-33.

$[\mathrm{LR}]_{1}$ R. Levy and M. D. Rice, The approximation and extension of uniformly continuous mappings, Fifth Prague Topological Symposium 1981, Heldermann-Verlag, Berlin, 1982, 455-461. 
$[\mathrm{LR}]_{2} \longrightarrow$, Techniques and examples in $U$-embedding, submitted to Top. and Its Appl. preprint.

$[\mathrm{LR}]_{3} \ldots$, The extension of uniformly continuous Banach space-valued mappings, Proc. Special Session on Rings of Continuous Functions, Annual Meeting, American Mathematical Society, January, 1982, to be published by Marcel Dekker.

$[\mathrm{LR}]_{4} \ldots$, The approximation and extension of uniformly continuous Banach space-valued mappings, Comm. Math. Univ. Carolinae, 24, 2 (1983), 251-265.

Received April 8, 1983 and in revised form June 27, 1983.

GEORGE MASON UNIVERSITY

FAIRFAX, VA 22030 



\section{PACIFIC JOURNAL OF MATHEMATICS EDITORS}

DONALD BABBITT (Managing Editor)
University of California
Los Angeles, CA 90024
CHARLES R. DePrima
California Institute of Technology
Pasadena, CA 91125
R. FinN
Stanford University
Stanford, CA 94305

HERMANN FLASCHKA

University of Arizona

Tucson, AZ 85721

RAMESH A. GANGOLLI

University of Washington

Seattle, WA 98195

ROBION KIRBY

University of California

Berkeley, CA 94720

C. C. MOore

University of California

Berkeley, CA 94720
Hugo RossI

University of Utah

Salt Lake City, UT 84112

H. SAMELSON

Stanford University

Stanford, CA 94305

HaRold STARK

University of California, San Diego

La Jolla, CA 92093

\section{ASSOCIATE EDITORS}
R. ARENS
E. F. BECKENBACH (1906-1982)
B. H. NEUMANN
F. WOLF
K. YoshidA

\section{SUPPORTING INSTITUTIONS}

UNIVERSITY OF ARIZONA

UNIVERSITY OF BRITISH COLUMBIA

CALIFORNIA INSTITUTE OF TECHNOLOGY

UNIVERSITY OF CALIFORNIA

MONTANA STATE UNIVERSITY

UNIVERSITY OF NEVADA, RENO

NEW MEXICO STATE UNIVERSITY

OREGON STATE UNIVERSITY
UNIVERSITY OF OREGON

UNIVERSITY OF SOUTHERN CALIFORNIA

STANFORD UNIVERSITY

UNIVERSITY OF HAWAII

UNIVERSITY OF TOKYO

UNIVERSITY OF UTAH

WASHINGTON STATE UNIVERSITY

UNIVERSITY OF WASHINGTON 


\section{Pacific Journal of Mathematics}

\section{Vol. 117, No. $1 \quad$ January, 1985}

Amos Altshuler and Leon Steinberg, The complete enumeration of the

4-polytopes and 3 -spheres with eight vertices $\ldots \ldots \ldots \ldots \ldots \ldots \ldots \ldots$

Michael James Beeson, The $6 \pi$ theorem about minimal surfaces . . . . . . . . 17

Jeffrey Lawrence Caruso and Stefan Waner, An approximation theorem

for equivariant loop spaces in the compact Lie case ...............27

Jo-Ann Deborah Cohen, Topologies on the quotient field of a Dedekind

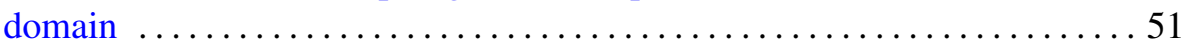

Szymon Dolecki, Gabriele H. Greco and Alois Andreas Lechicki,

Compactoid and compact filters .........................6 69

Roger William Hansell (Sr.), Generalized quotient maps that are

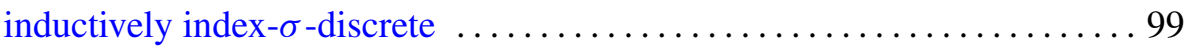

Gerhard Huisken, Capillary surfaces over obstacles $\ldots \ldots \ldots \ldots \ldots \ldots \ldots 121$

Jun Shung Hwang, A problem on continuous and periodic functions . . . . 143

Ronald Fred Levy and Michael David Rice, The extension of equi-uniformly continuous families of mappings $\ldots \ldots \ldots \ldots \ldots \ldots 149$

Kevin Mor McCrimmon, Derivations and Cayley derivations of generalized

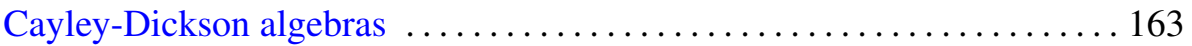

H. M. (Hari Mohan) Srivastava, A multilinear generating function for the Konhauser sets of biorthogonal polynomials suggested by the Laguerre

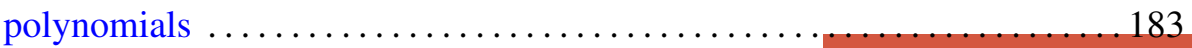

Zhu Jia Lu, Some maximum properties for a family of singular hyperbolic operators 